University of Nebraska - Lincoln

DigitalCommons@University of Nebraska - Lincoln

Faculty Publications, Department of Physics and Astronomy

Research Papers in Physics and Astronomy

2009

\title{
First-principles analysis of spin-disorder resistivity of Fe and $\mathrm{Ni}$
}

\author{
Aleksander L. Wysocki \\ University of Nebraska-Lincoln, awysocki@iastate.edu \\ Renat F. Sabirianov \\ University of Nebraska at Omaha, rsabirianov@mail.unomaha.edu \\ Mark van Schilfgaarde \\ Arizona State University, mark.van_schilfgaarde@kcl.ac.uk \\ Kirill D. Belashchenko \\ University of Nebraska-Lincoln, belashchenko@unl.edu
}

Follow this and additional works at: https://digitalcommons.unl.edu/physicsfacpub

Part of the Physics Commons

Wysocki, Aleksander L.; Sabirianov, Renat F.; van Schilfgaarde, Mark; and Belashchenko, Kirill D., "Firstprinciples analysis of spin-disorder resistivity of Fe and Ni" (2009). Faculty Publications, Department of Physics and Astronomy. 89.

https://digitalcommons.unl.edu/physicsfacpub/89

This Article is brought to you for free and open access by the Research Papers in Physics and Astronomy at DigitalCommons@University of Nebraska - Lincoln. It has been accepted for inclusion in Faculty Publications, Department of Physics and Astronomy by an authorized administrator of DigitalCommons@University of Nebraska Lincoln. 


\title{
First-principles analysis of spin-disorder resistivity of $\mathrm{Fe}$ and $\mathrm{Ni}$
}

\author{
A. L. Wysocki, ${ }^{1}$ R. F. Sabirianov, ${ }^{2}$ M. van Schilfgaarde, ${ }^{3}$ and K. D. Belashchenko ${ }^{1}$ \\ ${ }^{1}$ Department of Physics and Astronomy and Nebraska Center for Materials and Nanoscience, University of Nebraska-Lincoln, \\ Lincoln, Nebraska 68588, USA \\ ${ }^{2}$ Department of Physics and Nebraska Center for Materials and Nanoscience, University of Nebraska-Omaha, \\ Omaha, Nebraska 68182, USA \\ ${ }^{3}$ Department of Chemical and Materials Engineering, Arizona State University, Tempe, Arizona 85287, USA \\ (Received 11 May 2009; revised manuscript received 13 November 2009; published 23 December 2009)
}

\begin{abstract}
Spin-disorder resistivity of $\mathrm{Fe}$ and $\mathrm{Ni}$ and its temperature dependence are analyzed using noncollinear density functional calculations within the supercell method. Different models of thermal spin disorder are considered, including the mean-field approximation and the nearest-neighbor Heisenberg model. If the local moments are kept frozen at their zero-temperature values, very good agreement with experiment is obtained for $\mathrm{Fe}$ but for Ni the resistivity at elevated temperatures is significantly overestimated. Agreement with experiment for $\mathrm{Fe}$ is improved if the local moments are made self-consistent. The effect of short-range order on spindisorder resistivity is more pronounced in $\mathrm{Ni}$ compared to $\mathrm{Fe}$ but it is too weak to explain the overestimation of the resistivity for paramagnetic $\mathrm{Ni}$; the latter is therefore attributed to the reduction in the local moments down to $0.35 \mu_{B}$. Overall, the results suggest that low-energy spin fluctuations in Fe and Ni are better viewed as classical rotations of local moments rather than quantized spin fluctuations that would require an $(S$ $+1) / S$ correction.
\end{abstract}

DOI: $10.1103 /$ PhysRevB.80.224423

PACS number(s): 75.47.- $\mathrm{m}, 75.50 . \mathrm{Bb}$

\section{INTRODUCTION}

Electron scattering off of spin fluctuations in magnetic metals results in an "anomalous" contribution to electric resistivity. ${ }^{1-3}$ The analysis of this spin-disorder resistivity (SDR) is of interest because it can provide material-specific information on the character of spin fluctuations which is not easily accessible by other means. Scattering on spin disorder is also an important factor degrading the performance of magnetoresistive nanostructures in spintronic devices.

The magnitude of the spin-disorder contribution to resistivity is comparable to the phonon contribution near and above the Curie temperature $T_{c}{ }^{1}$ (This is because magnetic scattering amplitudes have no small parameter unless the exchange splitting is small compared to the bandwidth.) It is usually assumed that SDR is constant well above $T_{c}$. In this region Matthiessen's rule is valid and the phonon contribution can be fitted to the Bloch-Grüneisen formula. The excess resistivity in the whole temperature range may be attributed to spin disorder, ${ }^{4}$ although one may expect deviations from Matthiessen's rule at low temperatures where transport is carried by weakly interacting spin channels. ${ }^{5}$ In addition, it was argued that in some cases (such as Ni) spin disorder may change the character of states on the Fermi level and thereby appreciably change the phonon contribution itself. ${ }^{1,2}$

Many authors have studied SDR theoretically using the $s-d$ model Hamiltonian. ${ }^{6-9}$ In this approach the $3 d$ shells in transition metals (or $f$ shells in rare-earth metallic magnets) are assumed to be localized at atomic sites and partially filled, forming magnetic moments $\hat{\mathbf{S}}_{i}$ that are coupled to the current-carrying conduction electrons by exchange interaction $H_{s d}=-J_{s d} \sum_{i} \hat{\mathbf{s}}_{i} \hat{\mathbf{s}}_{i}$, where $J_{s d}$ is the local $s$ - $d$ exchange coupling constant and $\hat{\mathbf{s}}_{i}$ is the spin-density operator of the conduction electrons at site $i$. Thermal fluctuations of the $d$-electron spins generate an inhomogeneous exchange po- tential; in the Born approximation the SDR is then determined by the conduction-electron band structure and the spin-spin correlation functions of $d$-electron spins. ${ }^{9}$ If the scattering is approximated as being elastic, only equal-time connected spin correlators have to be considered. Further, if the mean-field approximation (MFA) is used for $3 d$ spin statistics, the SDR behaves as $\rho_{\text {mag }}(T)=\rho_{0}\left[1-M^{2}(T) / S(S+1)\right]$, where $M(T)=\langle\mathbf{S}(T)\rangle$ is the magnetization at temperature $T$ and $\rho_{0} \propto J_{s d}^{2} S(S+1) .{ }^{6}$ Note that above $T_{c}$ SDR is constant and equal to $\rho_{0}$. The shape of the Fermi surface of conduction electrons is immaterial to this prediction as long as the scattering is elastic. ${ }^{9}$

The effects of magnetic short-range order (MSRO) on SDR have also been investigated within the $s$ - $d$ model. ${ }^{7,8,10-15}$ This problem has attracted considerable attention in connection with a "bump" in the resistivity that is observed near $T_{c}$ in some magnetic metals (although it is usually quite small). ${ }^{1}$ The analysis of critical MSRO effects showed that a cusp may appear near $T_{c}$ due to long-wave critical fluctuations, ${ }^{7}$ although it should usually be strongly suppressed by finite mean-free path and cancellations due to Fermi surface integration. ${ }^{10}$ It was also found that the effect of MSRO and even its sign are sensitive to such details of the model as the conduction-band occupation and the form of the scattering (pseudo)potential. ${ }^{12-14}$

Although the $s$ - $d$ model provided physical insight into the mechanism of SDR, it suffers from serious limitations. First, the distinction between localized and conduction electrons is not justified in transition metals where $3 d$ electrons are itinerant and form the Fermi surface. Even if the current is dominated by light $s$-like bands that can be distinguished from heavy $d$-like bands, the relaxation rate is dominated by interband $(s-d)$ scattering. ${ }^{16}$ Second, at elevated temperatures the scattering potential generated by spin disorder is of the order of the exchange splitting, which is not small compared 
to the bandwidth. This raises the question of the validity of the Born approximation, which is usually made in model calculations. Third, the $s$ - $d$ model does not properly take into account the change in electronic structure due to disorder.

The first-principles approach to SDR is free from all these limitations and can be used for quantitative calculations of SDR. This is of particular interest for the theory of itinerant magnets because, as mentioned above, SDR depends on spin-spin correlation functions. Different theories of itinerant magnetism make conflicting predictions for such properties as the degree of MSRO, the mean-squared magnetic moment, and their temperature dependence; ${ }^{17-21}$ these quantities are quite hard to measure directly. By calculating SDR for a particular model of spin fluctuations and comparing the results with experiment, one can attempt to validate or rule out different spin-fluctuation models.

Earlier we have calculated the temperature dependence of SDR in $\mathrm{Fe}$ and $\mathrm{Ni}$ using supercell calculations within the tight-binding linear muffin-tin orbital (TB-LMTO) method using the mean-field distribution for spin-orientation statistics. $^{22}$ Good agreement with experiment was obtained for $\mathrm{Fe}$ but for paramagnetic $\mathrm{Ni}$ the SDR was found to be significantly overestimated. In this paper we analyze the temperature dependence of SDR for $\mathrm{Fe}$ and $\mathrm{Ni}$ in greater detail. We focus on noncritical behavior and consider the effects of magnetic ordering, MSRO, and local moment reduction. The accuracy of the calculations is checked by varying the basis set size; the effect of self-consistency of the atomic potentials is also considered.

\section{GENERAL APPROACH AND METHODS}

Our approach is based on noncollinear density functional theory (DFT). All the valence electrons are treated on the same footing while the scattering potentials are determined by the self-consistent electron charge and spin densities. We use the TB-LMTO method ${ }^{23}$ which represents the electronic density of the crystal as a superposition of overlapping atomic spheres; the electronic density inside each sphere is spherically symmetric. This method is known to work very well in close-packed materials, and it allows us to introduce spin disorder in various ways. In this work we used the rigid spin approximation which assumes that the spin density in each atomic sphere remains collinear while the spin densities of different atomic spheres become noncollinear at finite temperatures. In the simplest model the electron charge and spin densities in all atomic spheres are taken from the ground state and frozen while the directions of the spin moments in different spheres are randomized with the angular distribution function taken from MFA at the given temperature. This model is expected to work reasonably well for Fe which has a fairly stable local moment. ${ }^{17,24}$ In Sec. III we show that this is indeed the case; however, for Ni the paramagnetic SDR calculated in this way is about twice too large. In order to explain this discrepancy, the dependence of SDR on the degree of MSRO and on the magnitude of the local moment is studied in Secs. IV and V.

We use the supercell approach and calculate the areal conductance of a layer of spin-disordered metal FM(D) sand-

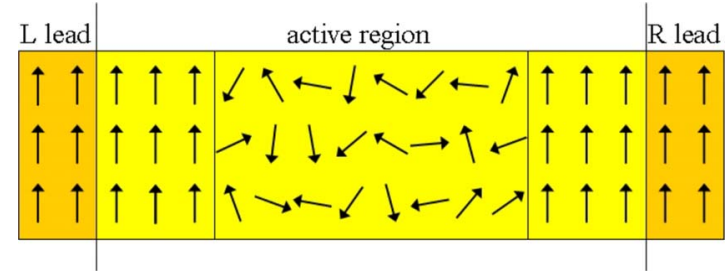

FIG. 1. (Color online) The schematic picture of the system used in the calculations. Vertical lines indicate the embedding planes.

wiched between fully ordered semi-infinite leads FM(O) made of the same metal (see Fig. 1). The resistivity is then proportional to the slope of the inverse conductance as a function of the disordered layer thickness, once the Ohmic limit is reached. For the given thickness of the FM(D) layer, the conductance of the system was averaged over several disorder configurations (typically 15). The planar system is represented by a laterally periodic prism with an axis along the [001] crystallographic direction, and care is taken to make sure that the conductance scales as the cross section of the prism. To calculate the conductance we use the principallayer Green's function technique ${ }^{25,26}$ and the LandauerBüttiker formalism ${ }^{27}$ in the implementation allowing for noncollinearity in the active region. ${ }^{28}$ This technique was employed before to study the effects of substitutional disorder on transport in magnetic multilayers $;{ }^{29}$ it is similar to the supercell Kubo-Greenwood method used to calculate the residual resistivity of binary alloys. ${ }^{30}$ We used experimental lattice constant both for Fe $(a=2.8665 \AA)$ and $\mathrm{Ni}(a=3.524 \AA)$. For $N \times N$ lateral supercell we used $60 / N \times 60 / N$ uniform $k$-point mesh for conductance calculations for both $\mathrm{Fe}$ and $\mathrm{Ni}$.

If the atomic potentials in the supercell are not converged to self-consistency with the given spin-disorder configuration, care needs to be taken to ensure local charge neutrality. Indeed, FM(D) and FM(O) materials have different Fermi levels that must normally be matched by the contact voltage. In order to enforce charge neutrality in the $\mathrm{FM}(\mathrm{D})$ region, a constant potential shift was introduced in this region so that the charge in the central part of FM(D) averaged over disorder realizations was zero. This (small) potential shift plays the role of the contact voltage. Note that no matter how the $\mathrm{FM}(\mathrm{O}) / \mathrm{FM}(\mathrm{D})$ interfaces are treated (self-consistently or not), they add contact resistances to the circuit. However, since the resistivity of the $\mathrm{FM}(\mathrm{D})$ material is extracted from the thickness dependence of the resistance in the Ohmic limit, the simplified treatment of interfaces has no effect on the results.

\section{SPIN-DISORDER RESISTIVITY IN THE MEAN-FIELD APPROXIMATION}

\section{A. Paramagnetic state}

In this section we analyze the temperature dependence of SDR for iron and nickel using MFA for thermal spin disorder; the connected spin-spin correlator is purely local in this approximation. First we consider the paramagnetic state 


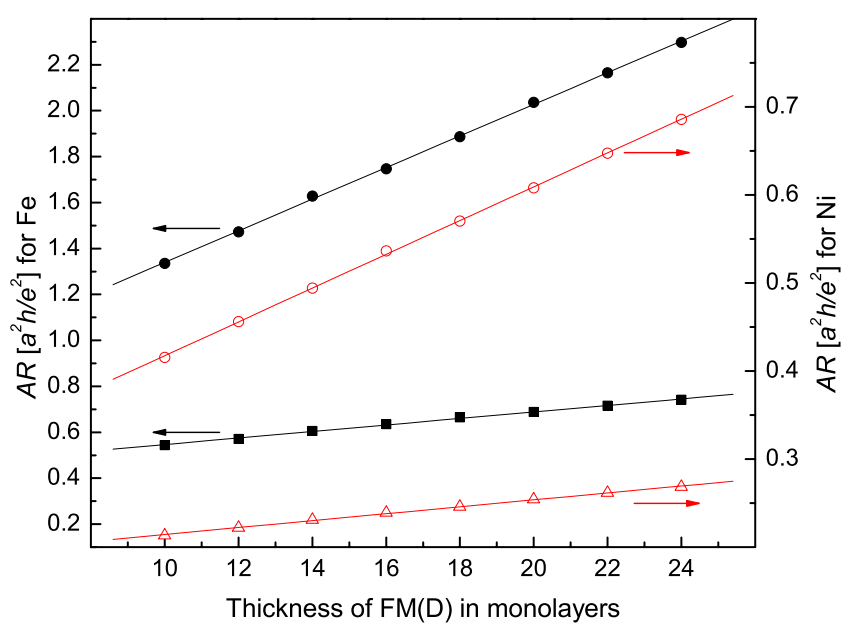

FIG. 2. (Color online) The area-resistance product $A R$ of the $\mathrm{FM}(\mathrm{O}) / \mathrm{FM}(\mathrm{D}) / \mathrm{FM}(\mathrm{O})$ systems as a function of the $\mathrm{FM}(\mathrm{D})$ layer thickness for bcc Fe (black filled symbols) and fcc Ni (red or gray empty symbols) obtained with $l_{\max }=2$. Circles and squares correspond, respectively, to the paramagnetic state and to the lowest temperature for which the calculations were made $\left(T=0.27 T_{c}\right.$ for $\mathrm{Fe}$ and $T=0.58 T_{c}$ for $\mathrm{Ni}$ ). $4 \times 4$ and $3 \times 3$ supercells were used for $\mathrm{Fe}$ and $\mathrm{Ni}$, respectively, with edges along the [100] directions. Straight lines show the linear fitting; error bars are smaller than the size of the symbols.

where the angular distribution function is isotropic and the resulting SDR is temperature independent.

We need to make a physically reasonable choice of atomic potentials for the conductance calculations. It is known that the local moments in $\mathrm{Fe}$ are quite stable; ${ }^{17}$ in particular, the DLM method, which employs the coherent-potential approximation for spin-disordered states, shows only a small reduction in the local moment in paramagnetic Fe compared to its ground-state value. ${ }^{24}$ As seen below, direct averaging of self-consistent local moments in the paramagnetic states gives a similar result. Therefore, for $\mathrm{Fe}$ it is reasonable to use frozen atomic potentials taken from the zero-temperature ground state in all calculations. We have also checked the effect of self-consistency on SDR in Fe and found it to be small (see below). The situation is entirely different for $\mathrm{Ni}$, where the local moment depends on the magnetic state; in particular, it vanishes altogether in the paramagnetic disordered local-moment (DLM) approximation. ${ }^{31}$ Since longitudinal spin fluctuations (that are absent in our approach) can at least partially restore the local moments, ${ }^{17}$ it is not a priori clear how the atomic potentials should be modified for $\mathrm{Ni}$. In this section we use frozen atomic potentials; the necessary corrections are discussed later.

Figure 2 shows the inverse areal conductance for paramagnetic $\mathrm{Fe}$ and $\mathrm{Ni}$ as a function of the disordered FM(D) region thickness. Here we used the frozen ground-state atomic potentials and the LMTO basis including $s, p$, and $d$ orbitals $\left(l_{\max }=2\right)$. The supercell cross sections contained $4 \times 4$ (for $\mathrm{Fe}$ ) and $3 \times 3$ (for $\mathrm{Ni}$ ) cubic unit cells with edges oriented along the [100] directions. Almost perfect Ohmic behavior is apparent for both $\mathrm{Fe}$ and $\mathrm{Ni}$, which establishes the validity of our approach.

Table I lists the values of SDR found for paramagnetic Fe
TABLE I. Spin-disorder resistivity in $\mu \Omega \mathrm{cm}$ for paramagnetic bcc Fe and fcc Ni. The calculated values are given for basis sets with $l_{\max }=2$ and 3 , as well as for different lateral cell sizes with edges along the [100] directions. SC denotes calculations with selfconsistent potentials. Standard deviations of SDR due to limited disorder sampling are included. The experimental value is from Ref. 4.

\begin{tabular}{lccccc}
\hline \hline & $M$ & & & & \\
Metal and basis & $\left(\mu_{B}\right)$ & $2 \times 2$ & $3 \times 3$ & $4 \times 4$ & Expt. \\
\hline Fe: $l_{\max }=2$ & 2.29 & $106 \pm 1.8$ & $101 \pm 1.3$ & $102 \pm 1.0$ & 80 \\
$l_{\max }=3$ & 2.22 & $86 \pm 1.6$ & $87 \pm 7.1$ & $85 \pm 7.4$ & 80 \\
$l_{\max }=2, \mathrm{SC}$ & 2.21 & $88 \pm 3.7$ & & & 80 \\
Ni: $l_{\max }=2$ & 0.66 & $34 \pm 0.6$ & $35 \pm 0.4$ & & 15 \\
$l_{\max }=3$ & 0.63 & $29 \pm 0.6$ & & & 15 \\
\hline \hline
\end{tabular}

and Ni using different supercell cross sections, LMTO bases truncated at $l_{\max }=2$ and $l_{\max }=3$ (the latter includes $f$ orbitals), as well as the value found using self-consistent (rather than frozen) atomic potentials for Fe. It is seen that the results are well converged with respect to the supercell cross section, and even $2 \times 2$ supercells provide sufficient accuracy. This is reasonable because the mean-free path in the paramagnetic state is short.

The calculations with self-consistent atomic potentials were performed as follows. In order to reduce the statistical error, the averaging of the conductance was performed using the same sets of random spin-disorder configurations as in the calculation with frozen potentials. For each individual spin configuration the atomic potentials were iterated to selfconsistency including the Fermi distribution function corresponding to the experimental $T_{c}$ of Fe. For $2 \times 2$ lateral cell for $\mathrm{Fe}$ we used $6 \times 6 \times 1 k$-point grid. The resulting distribution of the sites over the magnitude of the local magnetic moment is shown in Fig. 3; this distribution is Gaussian with a rather small width. The average local moment is only reduced by $3-4 \%$ from its ground-state value. This small reduction appears to be similar to the DLM calculations of Ref.

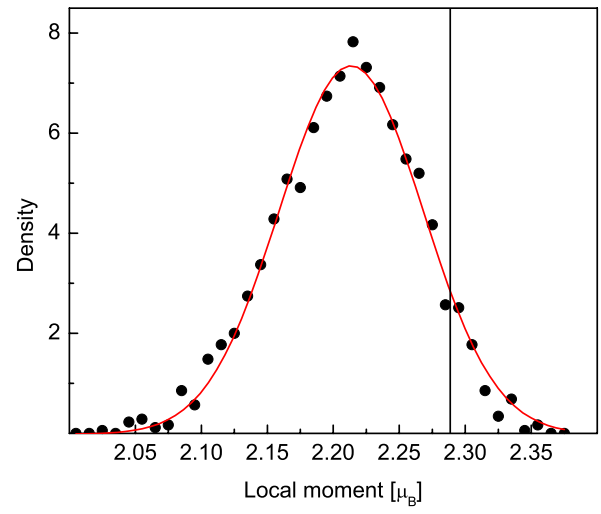

FIG. 3. (Color online) Distribution of the local magnetic moment in self-consistent fully spin-disordered bcc Fe. The Fermi temperature is equal to the experimental $T_{c}$. The vertical line shows the local moment at $T=0$. The red (solid) curve shows the Gaussian fit to the data. 


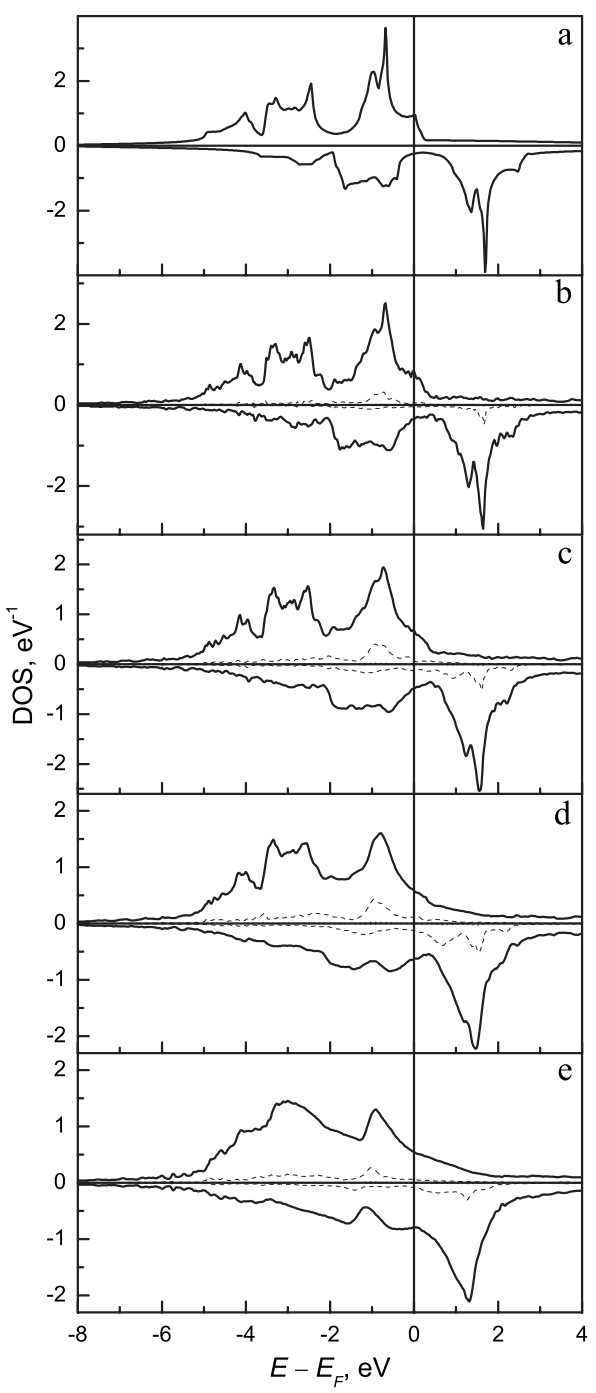

FIG. 4. Spin-resolved density of states (solid lines) for bcc Fe averaged over random spin configurations with the mean-field distribution function [Eq. (1)]; (a) $T=0$, (b) $T=0.25 T_{c}$, (c) $T=0.5 T_{c}$, (d) $T=0.75 T_{c}$, and (e) $T=T_{c}$. Dashed lines show the mean-square deviation of the DOS on a given site from its ensemble average.

20 while Ref. 24 obtained a somewhat larger reduction. The self-consistent density of states (DOS) (not shown) is very similar to the one generated by the frozen ground-state atomic potentials [see Fig. 4(e) below].

The addition of $f$ orbitals to the LMTO basis reduces the calculated SDR by approximately $15 \%$ for both Fe and Ni. Self-consistency in the paramagnetic state of Fe results in a similar reduction. This similarity suggests that the main reason for this SDR decrease is the reduction in the local moment, which is, incidentally, very similar in both cases. In order to check this, we performed additional calculations for $\mathrm{Fe}$ in which the $f$ channel was added to the basis while the charge density was kept unchanged from the self-consistent one with $l_{\max }=2$. For the frozen potential case, SDR reduced slightly from 106 to $100 \mu \Omega \mathrm{cm}$; for the self-consistent paramagnetic case, it only reduced from 88 to $86 \mu \Omega \mathrm{cm}$, which is within the error bar. Thus, the effect of increased $l_{\max }$ on SDR is mainly indirect through the local moment reduction. This is somewhat different from the binary alloy systems considered by other authors using both TB-LMTO and KKR (Korringa-Kohn-Rostocker) methods, where a larger effect of adding $f$ states was found. ${ }^{32,33}$ In view of the weak dependence of SDR on $l_{\max }$, below we use $l_{\max }=2$ in all calculations for $T<T_{c}$.

The experimental estimates of SDR in the paramagnetic state $^{4}$ are listed in the last column of Table I. The agreement with experiment for $\mathrm{Fe}$ is quite satisfactory, and it is in fact improved if the reduction in the local moment is included. In $\mathrm{Ni}$ the SDR calculated with frozen atomic potentials is overestimated by a factor of 2 . This is not surprising because, as mentioned above, the use of frozen atomic potentials is not justified for $\mathrm{Ni}$. In order to understand the origins of the disagreement with experiment for $\mathrm{Ni}$, possible modifications of the statistical model for the paramagnetic state must be considered; this is done below in Secs. IV and V.

Recently, Buruzs et al. ${ }^{34}$ calculated the SDR for $\mathrm{Fe}$ and Co using the DLM approach within the Korringa-KohnRostocker method and found that their method significantly overestimates the paramagnetic SDR in these metals. The source of disagreement with our supercell method for Fe is unknown to us.

\section{B. Temperature dependence in the ferromagnetic state}

In this section we consider the ferromagnetic state of $\mathrm{Fe}$ and Ni. We use frozen ground-state potentials and the basis with $l_{\max }=2$. As mentioned above, this approximation is reasonable for $\mathrm{Fe}$ while for $\mathrm{Ni}$ it is not applicable at high temperatures; nevertheless, comparison of these two systems will allow us to draw important conclusions. For the ferromagnetic state the spin configurations were generated using the mean-field distribution function,

$$
p(\theta) \propto e^{-\beta \mathbf{H}_{\mathrm{eff}} \boldsymbol{\mu}}, \quad H_{\mathrm{eff}}(T)=\frac{3 M(T) T_{c}}{\mu M(0)},
$$

where $\theta$ is the angle between the local moment $\boldsymbol{\mu}$ and the magnetization axis, $M(T)$ is the magnetization at temperature $T=(k \beta)^{-1}$ in MFA, and $\mathbf{H}_{\text {eff }}$ is the Weiss field. This distribution function depends only on $T / T_{c}$.

Before we turn to the temperature dependence of SDR, let us look at the electronic structure of $\mathrm{Fe}$ and $\mathrm{Ni}$ with spin disorder. The spin-resolved DOS of $\mathrm{Fe}$ and $\mathrm{Ni}$ is shown in Figs. 4 and 5 for several temperatures. These data were obtained by projecting the site-resolved DOS onto local spin-up and spin-down states (in the local reference frame where the $z$ axis is parallel to the local moment) and subsequent averaging over bulklike sites and spin-disorder configurations generated according to Eq. (1). The paramagnetic DOS of Fe is very similar to the KKR-DLM results. ${ }^{24}$ As the temperature is increased from 0 to $T_{c}$, the spin-up and spin-down states randomly hybridize with each other, the peaks broaden, and the van Hove singularities are washed out. The mean-squared deviation of the DOS from its average (shown by dashed lines) is quite small, which is a direct consequence of the large coordination number. In Fe the spin splitting is almost independent of temperature while in $\mathrm{Ni}$ it is much reduced as $T$ gets close to $T_{c}$. Note that the frozen atomic 


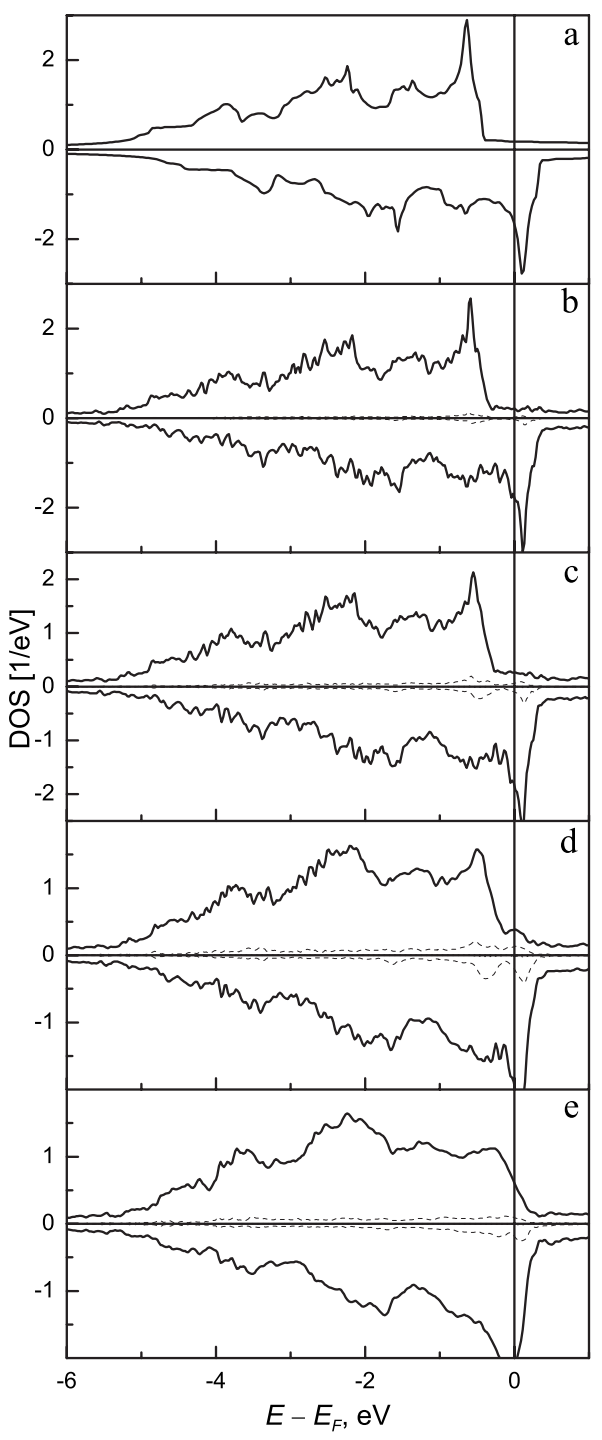

FIG. 5. Same as in Fig. 4 but for fcc Ni.

potentials in $\mathrm{Ni}$ are very far from self-consistency at elevated temperatures but a self-consistent treatment neglecting longitudinal spin fluctuations would be meaningless. We will return to this issue in Sec. V.

Let us now discuss the temperature dependence of SDR. While we found above that $2 \times 2$ supercells were sufficiently large for the paramagnetic state, additional care needs to be taken at lower temperatures where the mean-free path becomes longer. We found that $4 \times 4$ supercells for $\mathrm{Fe}$ and $3 \times 3$ for $\mathrm{Ni}$ demonstrate linear dependence of the arearesistance product on the length of the active region for all temperatures down to about $T_{c} / 3$ (see Fig. 2). This Ohmic behavior is consistent with a simple mean-free path estimate using the free-electron formula $l=\frac{3}{4} A R_{\mathrm{bal}} / \rho$, where $A R_{\mathrm{bal}}$ is the ballistic area-resistance product. According to this estimate, $l$ does not exceed the lateral cell size in this temperature range. An additional indication of the Ohmic behavior comes from the distribution of the current over the spin channels. The conductance of the $\mathrm{FM}(\mathrm{O}) / \mathrm{FM}(\mathrm{D}) / \mathrm{FM}(\mathrm{O})$ system is a sum of four partial conductances, $G_{\uparrow \uparrow}, G_{\downarrow \downarrow}, G_{\uparrow \downarrow}$, and $G_{\downarrow \uparrow}$ (the latter two are equal). Spin-conserving and spin-flip

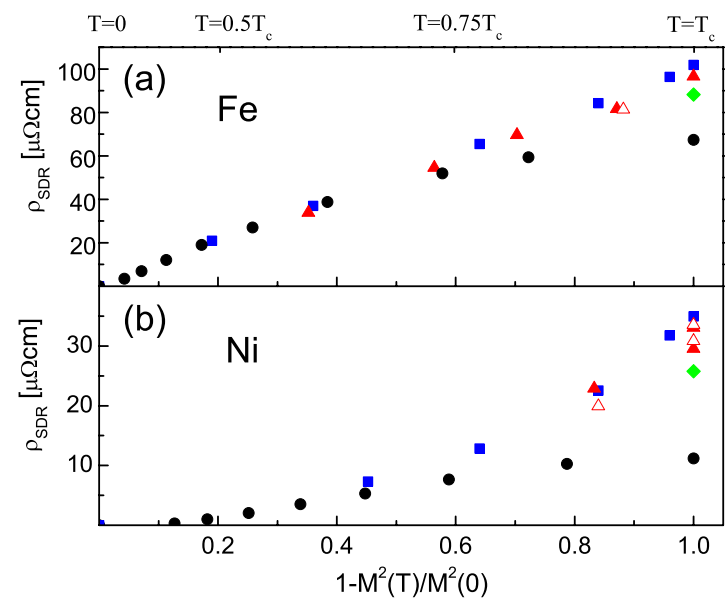

FIG. 6. (Color online) Dependence of spin-disorder resistivity on the magnetization for (a) Fe and (b) Ni. Black circles denote experimental data combining Ref. 4 for $\rho_{\text {mag }}(T)$ and Ref. 35 for $M(T)$. (Blue) squares show mean-field calculations, filled (red) triangles denote Monte Carlo results, and (green) diamonds show reverse Monte Carlo calculations. The empty (red) triangles show Monte Carlo results with larger cells: $6 \times 6$ for $\mathrm{Fe}$ and $4 \times 4$ for $\mathrm{Ni}$. The upper axis shows the MFA temperature corresponding to the given magnetization. All results are for $l_{\max }=2$. For MC results, $M(T)$ means the effective magnetization (see text).

scatterings have similar rates in our spin-disorder problem (as long as the temperature is not too low), and therefore the electrons "forget" their spin over their mean-free path. Therefore, in the Ohmic limit we should have $G_{\uparrow \uparrow} / G_{\uparrow \downarrow}=G_{\downarrow \uparrow} / G_{\downarrow \downarrow}$. This relation does indeed hold down to $T \sim T_{c} / 3$ unless the thicknesses of the FM(D) region is very small.

The dependence of the calculated SDR for Fe and Ni on the magnetization is plotted in Fig. 6 along with the experimental data ${ }^{4}$ [those for $M(T)$ were taken from Ref. 35]. The results for $\mathrm{Fe}$ agree rather well with experiment (see below for further analysis), especially at lower temperatures where the magnetic excitations are dominated by spin waves and our classical approach might be expected to be invalid. This surprising finding is due to the fact that SDR in Fig. 6 is plotted as a function of the long-range order parameter and that, as we show below in Sec. IV, the SDR in Fe is quite insensitive to MSRO. We expect that the temperature dependence of SDR should cross over to a distinct spin-wave regime at temperatures that are sufficiently low to restrict the scattering momentum transfer. Since the magnon dispersion is quadratic at low energies, $\omega=D q^{2}$ (where $D$ is the spinwave stiffness), and the typical scattering event involves $\hbar \omega \sim k T$, the maximum allowed momentum transfer $q_{\max } \propto \sqrt{T}$. Therefore, $q_{\max }$ becomes comparable to the size of the Fermi surface already at low temperatures, and the crossover temperature should be relatively low, consistent with our findings. The calculated SDR exhibits linear dependence on $M^{2}(T)$ up to $T_{c}$ while the experimental data deviate downward from the straight line. This deviation may be attributed to a small reduction in the local moment at elevated temperatures, as discussed in the previous section.

For Ni the deep low-temperature region could not be accessed due to the increased mean-free path. Still, the agree- 
ment with experiment at lower temperatures is good while at higher temperatures the calculated SDR increases too rapidly with $1-M^{2}(T) / M^{2}(0)$. This deviation indicates the inadequacy of our spin-fluctuation model; its possible modifications are studied in the following sections.

The qualitative features of the calculated temperature dependence of SDR (with frozen atomic potentials) are different for $\mathrm{Fe}$ and $\mathrm{Ni}$. If the spin fluctuations are treated classically within MFA and the scattering is treated perturbatively (within the Born approximation), the scattering potential scales in proportion to the local moment. These approximations applied to the $s$ - $d$ model predict that the SDR is proportional to $1-M^{2}(T) / M^{2}(0)$. It is seen in Fig. 6 that for $\mathrm{Fe}$ it is indeed proportional to $1-M^{2}(T) / M^{2}(0)$. However, this relation does not hold for $\mathrm{Ni}$. This indicates that the change in electronic structure owing to spin disorder, which the perturbative treatment does not include, has a large effect on SDR in Ni.

As seen in Figs. 4 and 5, the densities of states change quite appreciably with temperature for both $\mathrm{Fe}$ and $\mathrm{Ni}$. Therefore, it may seem surprising that for Fe the temperature dependence of SDR agrees with the $s$ - $d$ model. Still, one can understand the difference between $\mathrm{Fe}$ and Ni using the following considerations. First, the exchange splitting in $\mathrm{Ni}$ is strongly reduced at elevated temperatures (see Fig. 5); this reduction causes the heavy majority-spin $3 d$ bands to rise to the Fermi level. Scattering into these final states from the light bands becomes possible, which decreases the lifetime of the latter. This mechanism was invoked by $\mathrm{Mott}^{2}$ to argue that the reduction in the spin splitting in $\mathrm{Ni}$ can result in an anomalous temperature dependence of the phonon resistivity. The same argument applies to SDR considered here. According to Fig. 5, this happens approximately at $T=0.75 T_{c}$, which roughly corresponds to the upturn of SDR seen in Fig. 6(b). On the other hand, for Fe, as seen in Fig. 4, the exchange splitting is essentially constant, and no new bands are expected to appear at the Fermi level. Consequently, no additional temperature dependence is introduced and SDR scales as $1-M^{2}(T) / M^{2}(0)$.

While plausible, the above arguments are not conclusive because they assume without proof that the scattering matrix elements between the light and heavy bands are large. On a more subtle level, one may speculate that the difference between $\mathrm{Fe}$ and $\mathrm{Ni}$ can be understood based on the relation between disorder broadening and spin splitting. At the given wave vector, the spectral function consists of delta-function peaks corresponding to majority- and minority-spin states. In the presence of spin disorder, the spin states on neighboring sites are allowed to hybridize with random matrix elements, and the delta-function peaks broaden. At low temperature the broadening is small, and the peaks corresponding to different spins are well separated in energy from each other. However, at higher temperatures some of these peaks can merge and form common, "virtual-crystal-like" bands. Calculations of the paramagnetic spectral functions using the DLM method indicate that in Fe the majority- and minority-spin states remain separated through large portions of the Fermi surface even above $T_{c}{ }^{36}$ On the other hand, in Ni the majority- and minority-spin states are mixed in the paramagnetic state. ${ }^{36}$ Therefore, at certain temperature below $T_{c}$ there is a cross- over from separated to mixed-spin bands. The lifetime is expected to decrease as the bands merge, which again explains the upturn of SDR from the straight line in Fig. 6(b).

\section{EFFECT OF MAGNETIC SHORT-RANGE ORDER}

As mentioned above, short-range order can sometimes have a significant effect on resistivity. In this section we analyze the effect of MSRO on SDR in Fe and Ni. In particular, it is important to check whether the MFA (which neglects MSRO) is responsible for the large disagreement with experiment in Ni found in Sec. III. This is especially interesting because strong MSRO in Ni has been suggested by some experiments ${ }^{17,37}$ and theories. ${ }^{18,19}$

We studied the effect of MSRO on SDR using the Monte Carlo (MC) method for the classical Heisenberg model with nearest-neighbor exchange interaction on bcc and fcc lattices (for $\mathrm{Fe}$ and $\mathrm{Ni}$, respectively). For a given temperature we built a MC supercell of the size of the disordered region $[\mathrm{FM}(\mathrm{D})]$. For Fe the lateral size of the $\mathrm{FM}(\mathrm{D})$ was usually $4 \times 4$ while for $\mathrm{Ni}$ we used both $3 \times 3$ and $4 \times 4$ lateral sizes (all measured in cubic unit cells). MC simulations were performed for these supercells using periodic boundary conditions and the standard Metropolis algorithm. ${ }^{38-40}$ The system was equilibrated (usually for $20000 \mathrm{MC}$ steps per site), and subsequently several spin configurations were produced (usually with a delay of 10000 steps per site between the snapshots). These spin configurations were then used to calculate SDR as described above. In order to reduce $\mathrm{FM}(\mathrm{O})$ / FM(D) interface resistance (and thus the variance of the calculated resistance), the spin configurations were uniformly rotated in spin space to make the total magnetic moment parallel to the ordered $\mathrm{FM}(\mathrm{O})$ regions. Similar to the case of MFA configurations, linear dependence of resistance on the thickness of FM(D) layer was observed.

In order to study the effect of MSRO on SDR we need to compare the results obtained in MFA with those from MC. This comparison is complicated by the fact that the resistivity calculations are only available for supercells of limited size. We would like to identify the effect of MSRO at the fixed magnetization but the latter is undefined for a system of finite size. One could use the root-mean-squared total magnetic moment as a substitute for the long-range order parameter as is commonly done in MC simulations ${ }^{38}$ but there is no reason to expect that the resistivity should depend on this quantity; it rather must depend on the connected spin correlators, which are also not known because they contain the subtracted term $M^{2}$. Moreover, since the root-mean-squared magnetization contains a statistical variance term scaling as $1 / N$, close to and above the thermodynamic $T_{c}$ it changes appreciably depending on the thickness of the FM(D) layer. Since the finite size of the supercell affects all statistical properties due to the corruption of the long-range fluctuations, one would not be justified in using the finite-sizescaled value of the magnetization for comparison with MFA results.

For these reasons, we adopt a different procedure. First, we notice that the set of all spin correlators $\left\langle\mathbf{e}_{0} \mathbf{e}_{n}\right\rangle$, where $\mathbf{e}_{n}=\mathbf{S}_{n} / S$, contains the same information as the set of all 
TABLE II. The effective temperatures $T_{\text {eff }}$ and the connected spin-spin correlators $C_{0 n}$ (up to the third neighbor shell) for $\mathrm{Fe}$ and $\mathrm{Ni}$ for the Monte Carlo and reverse Monte Carlo models. The values of SDR are compared with the corresponding MFA results. These values include the statistical uncertainties related to the limited disorder sampling.

\begin{tabular}{|c|c|c|c|c|c|c|c|c|}
\hline \multirow{2}{*}{$\begin{array}{l}\text { Metal, } \\
\text { cross section }\end{array}$} & \multirow[b]{2}{*}{$T / T_{c}$} & \multirow[b]{2}{*}{$T_{\mathrm{eff}} / T_{c}$} & \multicolumn{4}{|c|}{$C_{0 i}=\left\langle\mathbf{e}_{0} \mathbf{e}_{i}\right\rangle-m_{\mathrm{eff}}^{2}$} & \multicolumn{2}{|c|}{$\rho_{\mathrm{mag}}(\mu \Omega \mathrm{cm})$} \\
\hline & & & $i=0$ & $i=1$ & $i=2$ & $i=3$ & $\mathrm{MC}$ or $\mathrm{RMC}$ & MFA \\
\hline $\mathrm{Fe}, 4 \times 4$ & $\infty$ & $\infty$ & 1 & 0 & 0 & 0 & $101.9 \pm 1.0$ & $101.9 \pm 1.0$ \\
\hline $\mathrm{Fe}, 4 \times 4$ & 1.217 & 1.177 & 1.000 & 0.184 & 0.110 & 0.073 & $96.6 \pm 1.9$ & $101.9 \pm 1.0$ \\
\hline $\mathrm{Fe}, 4 \times 4$ & 0.974 & 0.958 & 0.871 & 0.206 & 0.144 & 0.111 & $81.6 \pm 2.1$ & $88.1 \pm 0.5$ \\
\hline $\mathrm{Fe}, 6 \times 6$ & 0.974 & 0.965 & 0.882 & 0.209 & 0.144 & 0.112 & $81.4 \pm 2.3$ & $88.9 \pm 0.5$ \\
\hline $\mathrm{Fe}, 4 \times 4$ & 0.852 & 0.848 & 0.703 & 0.156 & 0.107 & 0.079 & $69.6 \pm 1.8$ & $71.3 \pm 0.4$ \\
\hline $\mathrm{Fe}, 4 \times 4$ & 0.730 & 0.724 & 0.564 & 0.126 & 0.087 & 0.066 & $54.6 \pm 1.8$ & $57.5 \pm 0.3$ \\
\hline $\mathrm{Fe}, 4 \times 4$ & 0.487 & 0.482 & 0.352 & 0.083 & 0.059 & 0.045 & $33.8 \pm 0.7$ & $35.8 \pm 0.2$ \\
\hline $\mathrm{Fe}, 4 \times 4$ & $\mathrm{RMC}$ & $\mathrm{RMC}$ & 1.000 & 0.309 & 0.167 & 0.060 & $88.2 \pm 1.3$ & $101.9 \pm 1.0$ \\
\hline $\mathrm{Ni}, 3 \times 3$ & $\infty$ & $\infty$ & 1 & 0 & 0 & 0 & $34.9 \pm 0.4$ & $34.9 \pm 0.4$ \\
\hline $\mathrm{Ni}, 3 \times 3$ & 1.269 & 1.234 & 1.000 & 0.146 & 0.071 & 0.052 & $33.1 \pm 0.8$ & $34.9 \pm 0.4$ \\
\hline $\mathrm{Ni}, 4 \times 4$ & 1.269 & 1.262 & 1.000 & 0.140 & 0.064 & 0.045 & $33.6 \pm 0.7$ & $34.9 \pm 0.4$ \\
\hline $\mathrm{Ni}, 3 \times 3$ & 1.110 & 1.063 & 1.000 & 0.205 & 0.129 & 0.104 & $29.5 \pm 1.3$ & $34.9 \pm 0.4$ \\
\hline $\mathrm{Ni}, 4 \times 4$ & 1.110 & 1.089 & 1.000 & 0.190 & 0.110 & 0.087 & $30.8 \pm 1.2$ & $34.9 \pm 0.4$ \\
\hline $\mathrm{Ni}, 3 \times 3$ & 0.952 & 0.943 & 0.833 & 0.172 & 0.104 & 0.086 & $22.9 \pm 0.9$ & $22.4 \pm 0.6$ \\
\hline $\mathrm{Ni}, 4 \times 4$ & 0.952 & 0.947 & 0.841 & 0.175 & 0.106 & 0.085 & $19.9 \pm 1.3$ & $22.7 \pm 0.6$ \\
\hline $\mathrm{Ni}, 4 \times 4$ & $\mathrm{RMC}$ & $\mathrm{RMC}$ & 1.000 & 0.335 & 0.140 & 0.114 & $25.8 \pm 0.8$ & $34.9 \pm 0.4$ \\
\hline
\end{tabular}

connected correlators (because it contains the value of magnetization in its large-distance limit). However, it is clear that the cutoff at the mean-free path and the averaging over neighbor pairs and over the Fermi surface ${ }^{10}$ make the longdistance behavior of the correlation function irrelevant for the resistivity. Therefore, it is physically reasonable to assume that the resistivity should depend at most on the first few spin correlators. These correlators are directly computable in MC simulations, and moreover converge very quickly as the simulation cell is increased. In particular, they are almost independent of the third dimension of the supercell with the given cross section.

Under the assumption that the first few correlators completely determine the resistivity, we proceed to establish a correspondence between the given finite-size supercell and an infinite system with the same Hamiltonian but at a slightly different temperature in such a way that several first correlators are matched. In practice, we tabulated the temperature dependence of the first three spin correlators for a large $12 \times 12 \times 12$ supercell; the correlators are well converged for all considered temperatures for this cell size. In spite of the relatively small size of the supercells used in resistivity calculations, the correlators found in MC simulations for these supercells were found to be almost but not quite converged to the tabulated infinite-lattice values. Naturally, the deviations get larger in the immediate proximity of $T_{c}$. Importantly, although the correlators vary slightly as a function of the supercell length (at the fixed cross section), this variation does not contain a systematic trend and usually converges rather quickly. We also found that in the range of supercell aspect ratios used in our calculations there are no signs of a crossover to an effectively one-dimensional behavior. Such a crossover is clearly observed in the magnetization curve but it sets in at a larger aspect ratio; our results are therefore free of the corresponding artifacts.

We then find the effective temperature at which the exact tabulated first nearest-neighbor correlator matches its value for the given supercell cross section averaged over its third dimension (length). We found that the second and third correlators automatically match with the tabulated values very accurately, which lends more credence to the whole procedure. The effective temperature is somewhat smaller than the actual one, the difference decreasing quickly with the increasing size of the supercell (see Table II). Finally, we use the magnetization in the thermodynamic limit at the effective temperature for comparison with MFA results. To find this magnetization, we first find $T_{c}$ using the fourth-order cumulant method. ${ }^{39}$ If the effective temperature is above $T_{c}$, the magnetization is set to zero; otherwise it is found using finite-size scaling. Since we are away from the critical region, we assumed in most cases a simple scaling form $M_{L}^{2}(T) / M(0)=M_{\infty}^{2}(T) / M(0)+A / N$, where $N$ is a number of atoms and $A$ is some constant. Using $L=8,10,12,14$ for both bcc and fcc lattices we found that this scaling form holds. The magnetization defined in this way can be called the effective magnetization.

Thanks to this procedure we are able to avoid difficult issues related to the analysis of critical behavior in our finitesize supercells. Our results represent the relation between the magnetic state and the resistivity, and not the magnetic thermodynamics. In this sense, they are more general than the particular thermodynamic model that is used to generate magnetic configurations.

The calculated SDR is shown in Fig. 6 as a function of the effective magnetization. In addition, the effective tempera- 
tures and the magnitude of MSRO can be inferred from Table II where the connected spin correlators for the first three shells of nearest neighbors are shown. They are calculated using the effective magnetization as well; therefore they correspond to the infinite system to which our finite-size spin ensembles are mapped.

For Fe the presence of MSRO leads only to a slight decrease in SDR. In order to verify the convergence with respect to the supercell size, we performed an additional calculations for $\mathrm{Fe}$ with a larger $6 \times 6$ supercells. In this case the effective temperature is almost equal to the actual temperature, the root-mean-squared magnetization does not depend strongly on the FM(D) thickness, and it is closer to the effective magnetization. The calculated SDR is shown as an empty triangle in Fig. 6(a). We can see that the result is essentially identical to the one obtained using $4 \times 4$ supercells, proving that it is already converged.

For Ni the presence of MSRO also leads to a decrease in SDR which, while still relatively small, is noticeably larger than for Fe. Several points at $M=0$ represent different calculations for the paramagnetic state; the corresponding effective temperatures can be found in Table II. Note that in general MSRO in the nearest-neighbor Heisenberg model is weaker for fcc lattice than for bcc (see Table II) due to larger coordination number of fcc lattice. Clearly, the effect of MSRO on SDR is significantly stronger in Ni compared to $\mathrm{Fe}$.

These results show that MSRO characteristic for the nearest-neighbor Heisenberg model has an almost negligible effect on SDR in Fe, and a more notable but still relatively small SDR reduction in Ni. While MSRO in the nearestneighbor Heisenberg model for the close-packed lattices considered here is not strong, it is seen that its effect on SDR is much smaller even compared with the values of the nearestneighbor spin-spin correlators. This insensitivity is likely due to the averaging over all the electronic states on the Fermi surface, ${ }^{10}$ which should be very effective in destroying the interference from scattering at different sites in transition metals with complicated Fermi surfaces. In fact, this averaging is also responsible for the small standard deviation of the local DOS from its mean (Fig. 4) and justifies the DLM approach for transition metals.

The spin-spin correlation function in real materials may be more complicated than in the nearest-neighbor Heisenberg model. However, if the interaction has a longer range while remaining mainly ferromagnetic, the MSRO must be weaker compared to the nearest-neighbor model. ${ }^{41}$ Firstprinciples calculations for both ferromagnetic and paramagnetic nickel show that the exchange parameters beyond nearest neighbors while being much smaller than the dominant nearest-neighbor exchange, stay mainly ferromagnetic. ${ }^{20,42}$ Interaction of this kind cannot support stronger MSRO compared to the nearest-neighbor Heisenberg model.

Nevertheless it is of interest to study whether MSRO that is stronger than in nearest-neighbor Heisenberg model can have more pronounced effect on SDR. For this purpose we used the reverse Monte Carlo (RMC) method $^{43}$ to produce a set of spin configurations with zero magnetization and deliberately targeting strong MSRO for the nearest neighbors. Due to geometrical constraints, the spin-spin correlators in different neighbor shells are not independent. We found it quite difficult to produce strongly correlated nearest neighbors and at the same time avoid unphysical artifacts in the long-range behavior of the correlation function. The spinspin correlators for the first three shells of neighbors in our RMC model are listed in Table II. The corresponding values of SDR calculated for $\mathrm{Fe}$ and $\mathrm{Ni}$ with this set of spin configurations are also listed in Table II and shown by full and empty diamonds in Fig. 6 . Here we used $4 \times 4$ supercells for both $\mathrm{Fe}$ and $\mathrm{Ni}$ and checked for finite-size effects using $6 \times 6$ supercells for $\mathrm{Fe}$ (essentially no difference was observed compared to $4 \times 4$ cells). As seen in Table II, the MSRO in this model is significantly stronger compared to the nearest-neighbor Heisenberg model. The effect of this strong MSRO leads to larger decrease in SDR as compared with MC results but it is still relatively small; the SDR is reduced compared to its MFA values by $12 \%$ for $\mathrm{Fe}$ and $22 \%$ for $\mathrm{Ni}$.

\section{EFFECT OF THE LOCAL MOMENT REDUCTION}

Reduction in the local moment is a universal feature of itinerant magnets as revealed by spin-fluctuation theories. ${ }^{17}$ As discussed in Sec. III, the local moment in Fe is very stable and changes only slightly in the paramagnetic state compared to zero temperature. Therefore, our calculations based on the ground-state value of the local moment agree well with experiment for Fe. Still, the SDR is sensitive to the local moment, and a small reduction in it noticeably improved the agreement with experiment at higher temperatures. Since the SDR was found to be insensitive to MSRO, it is reasonable to attribute the large overestimation of the high-temperature SDR in $\mathrm{Ni}$ to the neglect of the localmoment reduction. Here we study this issue in detail.

In the paramagnetic DLM state the local moment in $\mathrm{Ni}$ vanishes $^{31}$ but it is partially restored by longitudinal spin fluctuations. ${ }^{17,20}$ Following the idea of separation of low- and high-energy fluctuations, we assume that the current-carrying quasiparticles near the Fermi level experience the averaged exchange-correlation field generated by fast longitudinal spin fluctuations, and that this "mean field" is adequately represented by noncollinear DFT with disordered local moments constrained to their square-averaged values. The atomic potentials are therefore obtained using the fixed spin method ${ }^{44}$ with the value of the constrained local moment treated as an adjustable parameter, which has a physical meaning and can be measured experimentally. Other approximations are, in principle, possible; for example, the longitudinal spin fluctuations can be explicitly included in the same noncollinear DFT approach, i.e., they can be considered to be "slow" rather than "fast." Since the separation in slow and fast degrees of freedom is not well defined, we did not attempt to study the role of these additional fluctuations.

The calculated paramagnetic SDR of $\mathrm{Ni}$ as a function of the local moment is shown in Table III. As seen, SDR is very sensitive to the value of the local moment. Comparison with experimental SDR shows that our predicted value of the square-averaged local moment in paramagnetic state of $\mathrm{Ni}$ is equal to $0.35 \mu_{B}$ (using the more accurate basis set with 
TABLE III. Spin-disorder resistivity in $\mu \Omega \mathrm{cm}$ for paramagnetic $\mathrm{Ni}$ as a function of the fixed local moment. $2 \times 2$ supercells and basis sets with $l_{\max }=2$ and $l_{\max }=3$ were used. Standard deviations of SDR due to limited disorder sampling are included. The experimental value is $15 \mu \Omega \mathrm{cm}$ (Ref. 4).

\begin{tabular}{lcccc}
\hline \hline Local moment $\left(\mu_{B}\right)$ & 0.66 & 0.5 & 0.4 & 0.3 \\
\hline$l_{\max }=2$ & $34 \pm 0.6$ & $27 \pm 0.5$ & $21 \pm 0.4$ & \\
$l_{\max }=3$ & $29 \pm 0.6^{\mathrm{a}}$ & $23 \pm 0.5$ & $18 \pm 0.4$ & $12 \pm 0.3$ \\
\hline \hline
\end{tabular}

aThis value corresponds to unconstrained local moment of $0.63 \mu_{B}$.

$l_{\text {max }}=3$ ). Unfortunately, we are not aware of experimental measurements suitable for comparison with this prediction.

\section{DISCUSSION AND CONCLUSIONS}

Numerous previous studies ${ }^{6-10}$ based on the $s$ - $d$ model concluded that SDR in the paramagnetic state is essentially proportional to $J_{s d}^{2} S(S+1)$, where $S$ is the spin of the partially filled $3 d$ shell. This dependence is easy to understand based on the Fermi golden rule with averaging over the initial and final states of the $3 d$ spin but it assumes with no justification that the local $3 d$ spin is a good quantum number. In our treatment based on noncollinear DFT, the exchangecorrelation field with randomized directions on different sites plays the role of the $s-d$ Hamiltonian. However, contrary to the $s$ - $d$ model, the $3 d$ spin is treated classically, i.e., $\mathbf{S}$ is just a classical vector and not an operator. The Fermi golden rule in our case would give a paramagnetic SDR proportional to $J_{s d}^{2} S^{2}$. Thus, if the $S(S+1)$ factor were correct, noncollinear DFT calculations would underestimate the paramagnetic SDR by a factor $(S+1) / S$. This factor is close to 2 for $\mathrm{Fe}$ and more than 3 for Ni. In reality, the calculated SDR agrees well with experiment for $\mathrm{Fe}$ and is overestimated for $\mathrm{Ni}$ (if the local moment reduction is not included). We believe that these results provide clear evidence against the $S(S+1)$ factor which appears if the local moments are treated as localized atomic spins. Instead, the classical description of the local magnetic fluctuations in the spirit of the DLM approach is supported by our results. We suggest that the itinerancy of the $3 d$ electrons is crucial for this behavior. Qualitatively, one can argue that the low-energy fluctuations in $\mathrm{Fe}$ or $\mathrm{Ni}$ on the scale of $k T$ (which the resistivity is most sensitive to) are similar to classical rotations of the local moments rather than quantum fluctuations of localized spins. It would be interesting to investigate this issue for magnets with a varying degree of localization, including rare-earth systems.

Some poorly controlled assumptions are involved in the extraction of $\rho_{\mathrm{mag}}$ from the experimental data. ${ }^{4}$ First, it is assumed that $\rho_{\text {mag }}$ is constant in the wide temperature range above $T_{c}$, where the total resistivity is linear in $T$. This assumption implies that the local moments (or at least their mean-squared average) are constant in this range. Spinfluctuation theories for itinerant metals show that the local moments may change with temperature above $T_{c} \cdot{ }^{17,20,21}$ Such change will contribute to the slope of $\rho$ above $T_{c}$, and hence the separation of $\rho_{\text {mag }}$ from the phonon contribution would be inaccurate.

On the other hand, it has been argued that the phonon contribution to the resistivity may be sensitive to spin disorder because the latter may change the character of states at the Fermi level. ${ }^{1,2}$ In particular, in Ni the filled majority-spin $d$ states may be lifted up to the Fermi level by spin disorder, thereby facilitating interband $s-d$ scattering by phonons. This effect may therefore introduce an unusual temperature dependence of the phonon contribution, which makes spin disorder and phonon effects nonadditive, even if the scattering rates themselves obey Matthiessen's rule. Since we have not studied this effect here, our comparison of SDR with experiment for $\mathrm{Ni}$ is incomplete. However, the phonon contribution can be expected to follow the Bloch-Grüneisen form above $T_{c}$ with the electron-phonon scattering renormalized by spin disorder; therefore, the influence of spin disorder on the phonon contribution should not invalidate the procedure used for subtracting this contribution above $T_{c}$.

In conclusion, we have calculated the spin-disorder resistivity of $\mathrm{Fe}$ and $\mathrm{Ni}$ in the whole temperature range up to $T_{c}$. The role of MSRO was explored by comparing the resistivity for uncorrelated spin ensembles (mean-field approximation) with correlated spin configurations corresponding to the nearest-neighbor Heisenberg model. We found that the presence of MSRO leads to a reduction in SDR in both $\mathrm{Fe}$ and $\mathrm{Ni}$ while almost negligible in Fe, this effect is quite noticeable in Ni. The SDR in Fe depends linearly on $M^{2}(T)$ which implies that the main effect of spin disorder is to introduce scattering, which is proportional to the variance of the random potential. For Ni the calculated temperature dependence is more complicated; at elevated temperatures close to $T_{c}$ the SDR grows faster than expected. This faster increase in SDR may be explained by the reduction in the exchange splitting which lifts the heavy bands up to the Fermi level, thereby increasing the scattering rate. The results for $\mathrm{Fe}$ are in very good agreement with experiment if the atomic potentials are taken from zero temperature and frozen but for Ni the SDR calculated in this way is strongly overestimated. This disagreement is attributed to the reduction in the local magnetic moment in Ni. Comparison with experimental SDR leads to a value of $0.35 \mu_{B}$ above $T_{c}$, which may be compared with experiment.

\section{ACKNOWLEDGMENTS}

We are grateful to V. P. Antropov and E. Y. Tsymbal for useful discussions. This work was supported by the Nebraska Research Initiative and by the National Science Foundation through the Materials Science Research and Engineering Center (NSF-DMR Grant No. 0820521). K.D.B. is a Cottrell Scholar of Research Corporation. The work was completed utilizing the Research Computing Facility of the University of Nebraska-Lincoln. A portion of this research at Oak Ridge National Laboratory's Center for Nanophase Materials Sciences was sponsored by the Scientific User Facilities Division, Office of Basic Energy Sciences, U.S. Department of Energy. 
${ }^{1}$ B. R. Coles, Adv. Phys. 7, 40 (1958).

${ }^{2}$ N. F. Mott, Adv. Phys. 13, 325 (1964).

${ }^{3}$ S. V. Vonsovskii, Magnetism (Halsted, New York, 1974).

${ }^{4}$ R. J. Weiss and A. S. Marotta, J. Phys. Chem. Solids 9, 302 (1959).

${ }^{5}$ A. Fert and I. A. Campbell, Phys. Rev. Lett. 21, 1190 (1968); J. Phys. F: Met. Phys. 6, 849 (1976).

${ }^{6}$ T. Kasuya, Prog. Theor. Phys. 16, 58 (1956).

${ }^{7}$ P. G. de Gennes and J. Friedel, J. Phys. Chem. Solids 4, 71 (1958).

${ }^{8}$ I. Mannari, Prog. Theor. Phys. 26, 51 (1961).

${ }^{9}$ S. V. Vonsovskiı̆ and Yu. Izyumov, Sov. Phys. Usp. 5, 547 (1963).

${ }^{10}$ M. E. Fisher and J. S. Langer, Phys. Rev. Lett. 20, 665 (1968).

${ }^{11}$ J. B. Gibson, J. Phys. Chem. Solids 1, 27 (1956).

${ }^{12}$ P. L. Rossiter and P. Wells, J. Phys. C 4, 354 (1971).

${ }^{13}$ S. Alexander, J. S. Helman, and I. Balberg, Phys. Rev. B 13, 304 (1976).

${ }^{14}$ M. Kataoka, Phys. Rev. B 63, 134435 (2001).

${ }^{15}$ K. Akabli and H. T. Diep, J. Appl. Phys. 103, 07 F307 (2008).

${ }^{16}$ D. A. Goodings, Phys. Rev. 132, 542 (1963).

${ }^{17}$ T. Moriya, Spin Fluctuations in Itinerant Electron Magnetism (Springer, Berlin, 1985).

${ }^{18}$ V. P. Antropov, Phys. Rev. B 72, 140406(R) (2005).

${ }^{19}$ C. S. Wang, R. E. Prange, and V. Korenman, Phys. Rev. B 25, 5766 (1982).

${ }^{20}$ A. V. Ruban, S. Khmelevskyi, P. Mohn, and B. Johansson, Phys. Rev. B 75, 054402 (2007).

${ }^{21}$ A. L. Wysocki, J. K. Glasbrenner, and K. D. Belashchenko, Phys. Rev. B 78, 184419 (2008).

${ }^{22}$ A. L. Wysocki, K. D. Belashchenko, J. P. Velev and M. van Schilfgaarde, J. Appl. Phys. 101, 09G506 (2007).

${ }^{23}$ O. K. Andersen, Phys. Rev. B 12, 3060 (1975).

${ }^{24}$ B. L. Gyorffy, A. J. Pindor, J. Staunton, G. M. Stocks, and H. Winter, J. Phys. F: Met. Phys. 15, 1337 (1985).

${ }^{25}$ I. Turek, V. Drchal, J. Kudrnovský, M. Šob, and P. Weinberger, Electronic Structure of Disordered Alloys, Surfaces and Interfaces (Kluwer, Boston, 1997).
${ }^{26}$ J. Kudrnovský, V. Drchal, C. Blaas, P. Weinberger, I. Turek, and P. Bruno, Phys. Rev. B 62, 15084 (2000).

${ }^{27}$ S. Datta, Electronic Transport in Mezoscopic Systems (Cambridge University Press, Cambridge, 1995).

${ }^{28}$ M. van Schilfgaarde (unpublished).

${ }^{29}$ V. Drchal, J. Kudrnovský, P. Bruno, P. H. Dederichs, I. Turek, and P. Weinberger, Phys. Rev. B 65, 214414 (2002).

${ }^{30}$ R. H. Brown, P. B. Allen, D. M. Nicholson, and W. H. Butler, Phys. Rev. Lett. 62, 661 (1989).

${ }^{31}$ J. B. Staunton and B. L. Gyorffy, Phys. Rev. Lett. 69, 371 (1992).

${ }^{32}$ I. Turek, J. Kudrnovský, V. Drchal, L. Szunyogh, and P. Weinberger, Phys. Rev. B 65, 125101 (2002).

${ }^{33}$ J. Banhart, H. Ebert, P. Weinberger, and J. Voitländer, Phys. Rev. B 50, 2104 (1994).

${ }^{34}$ Á. Buruzs, L. Szunyogh, and P. Weinberger, Philos. Mag. 88, 2615 (2008).

${ }^{35}$ J. Crangle and G. M. Goodman, Proc. R. Soc. London 321, 477 (1971).

${ }^{36}$ J. Staunton, B. L. Gyorffy, A. J. Pindor, G. M. Stocks, and H. Winter, J. Phys. F: Met. Phys. 15, 1387 (1985).

${ }^{37}$ H. A. Mook, J. W. Lynch, and R. M. Nicklow, Phys. Rev. Lett. 30, 556 (1973).

${ }^{38}$ M. E. J. Newman and G. T. Barkema, Monte Carlo Methods in Statistical Physics (Clarendon, Oxford, 1999).

${ }^{39}$ D. P. Landau and K. Binder, A Guide to Monte Carlo Simulations in Statistical Physics (Cambridge University Press, Cambridge, 2000).

${ }^{40}$ K. Chen, A. M. Ferrenberg, and D. P. Landau, Phys. Rev. B 48, 3249 (1993).

${ }^{41}$ The MFA criterion of validity is $1 / z \ll 1$, where $z$ is the effective number of neighbors inside the interaction range.

${ }^{42}$ V. P. Antropov, B. N. Harmon, and A. N. Smirnov, J. Magn. Magn. Mater. 200, 148 (1999).

${ }^{43}$ R. L. McGreevy, J. Phys.: Condens. Matter 13, R877 (2001).

${ }^{44}$ V. L. Moruzzi, P. M. Marcus, K. Schwarz, and P. Mohn, Phys. Rev. B 34, 1784 (1986). 